\title{
Mastering the Skills Gap at Systemic Level - The Education Management Information System: A Key Element for an Effective Digital Transformation in Education
}

Daniel BÖJTE ${ }^{1}$

Abstract
Educational leaders must make informed predictions in order to design and
adopt relevant educational strategies to bridge the skills gap, so they need reliable and
trustworthy data. A well designed and properly rolled-out Education Management
Information System (EMIS) will assure precise, fluid and timely datasets which can be
easily interpreted, being able to extract the insights needed by using the adequate tools
for data analysis, filtering out redundant or irrelevant metrics.
Being not only an IT tool and a database, but rather a system within a larger
education system, EMIS contributes to implement the policies and practices by which
higher education institutions effectively collect, protect and use digital information assets
to meet academic and business needs.
This paper highlights the role and the importance of an effective EMIS and the
biggest challenges in implementing it.

Keywords: education management information system, EMIS, skills gap, digital transformation, education

JEL classification: I21, I23, I28, M15

DOI: $10.24818 /$ RMCI.2019.2.131

\section{Introduction}

The skills gap is a significant issue for countries worldwide, as it limits growth, in some cases, and increases the risks related to Industry 4.0 and automation. EMIS are countries' instruments for collecting, processing, and interpreting information about education and skills gaps. These systems have risen in complexity over the previous several centuries to offer policymakers and educators in the classroom, similar to an insight into whether learners, schools, and domestic education plans are moving towards clear goals. The systems are the cornerstone of educational reforms and tracking and assessment attempts (UNESCO, 2018).

The need to better match abilities and requirements on the workforce and to develop a more consistent and consistent system of qualifications is recognized as the main obstacles to competitiveness and economic development. Limited lifelong learning (LLL), vocational training (VET) and employment support facilities,

\footnotetext{
${ }^{1}$ Daniel Bőjte, Bucharest Academy of Economic Studies, Romania, E-mail: daniel.bojte@gmail.com
} 
particularly in the areas, have been found. Both a holistic vision of education connecting all levels and kinds of education and active intermediation and alignment between labor (schooling/training and LLL) and labor demand (development of economic and private sectors) is lacking, also made worse by the absence of anticipation and alignment of abilities.

EMIS is often regarded as a specialized unit responsible for collecting, compiling and disseminating data to decision-makers and for planning and reporting purposes, so that EMIS can be described as an institutional service unit that produces, manages and disseminates educational data and information for the purposes of collecting, storing, integrating, processing, organizing and producing (Hua and Herstein, 2003). Furthermore, an EMIS has a multi-faceted framework that includes technological and institutional provisions for information collection, processing, and dissemination in an educational scheme. It is crucial for monitoring changes, ensuring data quality and prompt reporting of significant planning and management information, and promoting the use of information by policymakers in decisionmaking. The efficacy of the system depends on its sustainability, accountability, and efficiency. An effective EMIS in planning and policy dialog is credible and operational. It is not just a database or a system for information technology (IT). People are a significant element of an EMIS, not only guiding the collection, maintenance, and dissemination of information but also leading the conversion of information into statistics used to inform decision-making. An EMIS is defined as a system that exists within the broader scheme of education (Abdul-Hamid, 2014). An Education Management System is a vital instrument which is at the core of the planning and policy implementation processes in a country's education system.

\section{EMIS as an evolving concept}

The General Assembly of the United Nations agreed in 2015 to pursue 17 Sustainable Development Goals (SDGs) in order to achieve peace and shared prosperity by 2030. SDG 4 specifically addresses the significance of education and commits nations to work towards 'inclusive and equitable quality education and promote lifelong learning opportunities for all.' SDG 4's expanded scope within the 2030 Agenda needs EMIS to go far beyond administrative information to information that can inform long-term instructional outcomes policies and plans. EMIS should also investigate the connection between academic results and other socio-economic development industries, such as poverty alleviation, health, and jobs. Also, while easy measurements like enrolment, attendance, and graduation demonstrate access and involvement in the schooling, they do not provide advice on the quality or significance of the schooling, how equitable and inclusive it is and what is being learned.

If breaking down occupations into their component operations can enhance our knowledge of workforce requirements and increase our awareness of what activities are required to perform specific tasks particularly well, could applying this kind of thinking to educational reform assistance enhance learning results? If we reframe the issue from a skills gap issue (generally related to vocational skills training) 
to a learning gap issue (related to enhanced cognitive and socio-emotional learning skills that are essential to employer-defined skill gaps), can we simultaneously reinforce life and livelihood trajectories? By reframing the issue as a result of a learning gap, we strive to connect education and jobs by distributing ownership and governance for the solution across the education and jobs industries.

The momentum to tackle the learning gap is swelling: U.N. Sustainable Development Goals highlight quality education and appropriate abilities for worldwide youth and adults as main tools to strengthen economies and reduce poverty. They provide a blueprint for future education - focused on accountability, resilience, relationship and creativity - and what the Brookings Institution rightly calls "range of abilities." While the objectives emphasize the need for behavioral abilities (literacy, mathematics, science) they also strengthen the socio-emotional abilities required to achieve behavioral acquisition communication. They also refer to life-long learning, beginning with early-year education through adulthood, and include topics such as good citizenship, involvement, sustainable development, and entrepreneurship. By breaking jobs into their component operations, we can enhance our knowledge of workforce requirements and increase our awareness of what activities are required to fulfill certain tasks. If we reframe the issue from a skills gap issue (generally related to vocational skills training) to a learning gap issue (related to enhanced cognitive and socio-emotional learning skills that are essential to employer-defined skill gaps), can we simultaneously reinforce life and livelihood trajectories? By reframing the issue as a result of a learning gap, we strive to connect education and jobs by distributing ownership and governance for the solution across the education and jobs industries.

The momentum to tackle the learning gap is swelling: U.N. Sustainable Development Goals highlight quality education and appropriate abilities for worldwide youth and adults as main tools to strengthen economies and reduce poverty. They provide a blueprint for future education - focused on accountability, resilience, relationship and creativity - and what the Brookings Institution rightly calls "range of abilities." While the objectives emphasize the need for behavioral abilities (literacy, mathematics, science) they also strengthen the socio-emotional abilities required to achieve behavioral acquisition communication. They also refer to life-long learning, beginning with early-year education through adulthood, and include topics such as good citizenship, involvement, sustainable development, and entrepreneurship. By breaking jobs into their component operations, we can enhance our knowledge of workforce requirements and increase our awareness of what activities are required to fulfill certain tasks. If we reframe the issue from a skills gap issue (generally related to vocational skills training) to a learning gap issue (related to enhanced cognitive and socio-emotional learning skills that are essential to employer-defined skill gaps), can we simultaneously reinforce life and livelihood trajectories? By reframing the issue as a result of a learning gap, we strive to connect education and jobs by distributing ownership and governance for the solution across the education and jobs industries.

The momentum to tackle the learning gap is swelling: U.N. Sustainable Development Goals highlight quality education and appropriate abilities for worldwide youth and adults as main tools to strengthen economies and reduce poverty. 
While the SDGs underscore the need for cognitive competencies (literacy, mathematics, science) they also reinforce the socio-emotional skills needed to succeed at the cognitive acquisition communication, critical thinking, problem solving, creativity, management of one's emotions. They also reference life-long learning, beginning with early-year education through adulthood, and include topics like good citizenship, involvement, sustainable development, and entrepreneurship.

In this context, there is a stringent need for a source of information which may provide both in-depth and birds-eye views on the skills, learning and knowledge gaps. Modern EMIS must be placed in a well-known location of a national education information system, covering other fields other than traditional ones, or at least technologically integrated with appropriate data sources, enabling learners to understand, for instance, who is spending what, whether they are genuinely learning what they need to understand and whether data disaggregation is feasible at college. Only these features will make an EMIS truly relevant to policy planning and education systems management (Montoya, 2018).

The development of EMIS must, therefore, go much further than the selection, operation, and updating of appropriate software. It must also include the right mix of vision, technical capabilities, human resources, and social procedures required to satisfy extended data requirements and provide superior information to decisionmakers operating in altering technological and policy environments. Hence, SDG 4 is a reason and cause for re-examining the data types used by EMIS ante-evaluating whether this information accurately corresponds to the requirements of the user spectrum, whether from a local, domestic, regional or global perspective. It is imperative not only to extend the coverage of instructional information to distinct kinds and levels of education and lifelong learning and to monitor integration and equity but also to evaluate and comprehend instructional information about other socio-economic growth vectors (UNESCO, 2018).

\section{The effectiveness of an EMIS system}

An EMIS enables to produce the following valued elements to enhance the quality of education (Abdul-Hamid, 2014):

- Data: In order to provide quality education, data and associated statistics on schooling are essential. "The achievement of good-quality education is dependent on the quality of statistics which inform the policy formulation, educational planning, management, and monitoring processes" (Makwati, Audinos, and Lairez, 2003). The presence of information and a system it produces understanding about the state of education in a nation to collect, retain, and report. However, there is still a lack in many nations of reliable, relevant, and readily available data on particular schools, educators, enrollment, and instructional results. Also, few nations have introduced quality assurance measures to monitor information gathered by their corresponding EMISs for quality, exactness, validity, reliability, and usefulness. 
- Efficient expenditure: Information systems allow nations to plan their education cost-effectively.

- Institutionalized data systems: An EMIS enables policymakers to manage an education system to generate quality outputs when institutionalized and driven by a clear vision and strategic planning. Unfortunately, many nations have spent resources in constructing information systems for education management, but often, these systems are not institutionalized, lack guidance, and are not integrated into strategic planning procedures.

- Data-driven policies: Information systems for education management are designed to assist public professionals in designing and implementing policies. Unfortunately, most nations have not developed policies on how EMIS information can be used in planning and decision-making. Even in nations with institutionalized information systems, they are hardly used to guide education strategies. (UNESCO, 2003)

- Smart investments: Investing smartly is one of the suggestions produced by the Education 2020 strategy of the World Bank. An EMIS's added value is that it empowers a decision-maker to create intelligent expenditure choices based on information and investment analytics that have been shown to contribute to learning (World Bank, 2011).

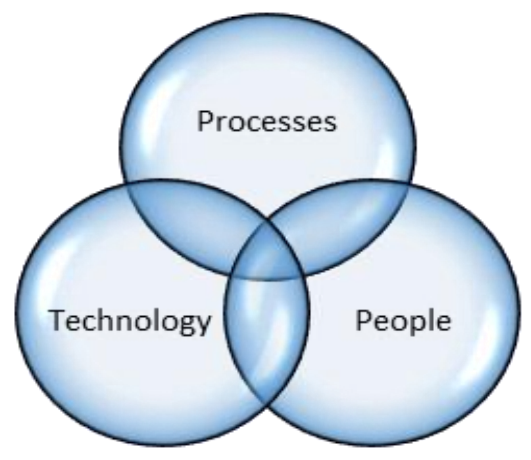

Figure 1 The components of an EMIS

Source: Author's own

Usually, EMIS aims to produce timely, cost-effective, and viable data that is reliable. A comprehensive education management information system aims to help integrate previously fragmented data from across subsectors, levels, and types of education. EMIS has been developing from the beginning "based on the idea that the Ministry of Education's (MoE's) information requirements needed to be considered from a wider perspective, one that involved acquiring both quantitative and qualitative information for educational policy, planning and implementation" (Wako, 2003).

A well-functioning system enables education stakeholders to answer a broad variety of issues. These problems range from planning and budgeting to problems related to staffing and leadership, institutional and system efficiency, education 
policies and strategies, and international reporting and collaboration. An extensive information management system for education should make it simpler for stakeholders to discover or demand data and resolve relationships and transitions between different kinds and educational levels. This often does not just mean going beyond the facts, but also analyzing, interpreting, and facilitating knowledge-sharing and policy dialogue.

An EMIS's success depends upon three factors (Hua and Herstein, 2003):

- Timeliness and reliability: Timely and reliable production of data and information

- Integration and sharing: Data integration and data sharing among departments

- Effective usage: Effective use of data and information for educational policy decisions.

Effective EMIS provides useful information for procedures of a) management and administration; b) policy formulation; c) tracking and assessment $=$ monitoring and evaluation. These procedures are interconnected and take place in educational systems, from central to local and within educational organizations themselves, at different places. (UNESCO, 2018)

Management
process

E-management portal

Data warehouse

Data sources
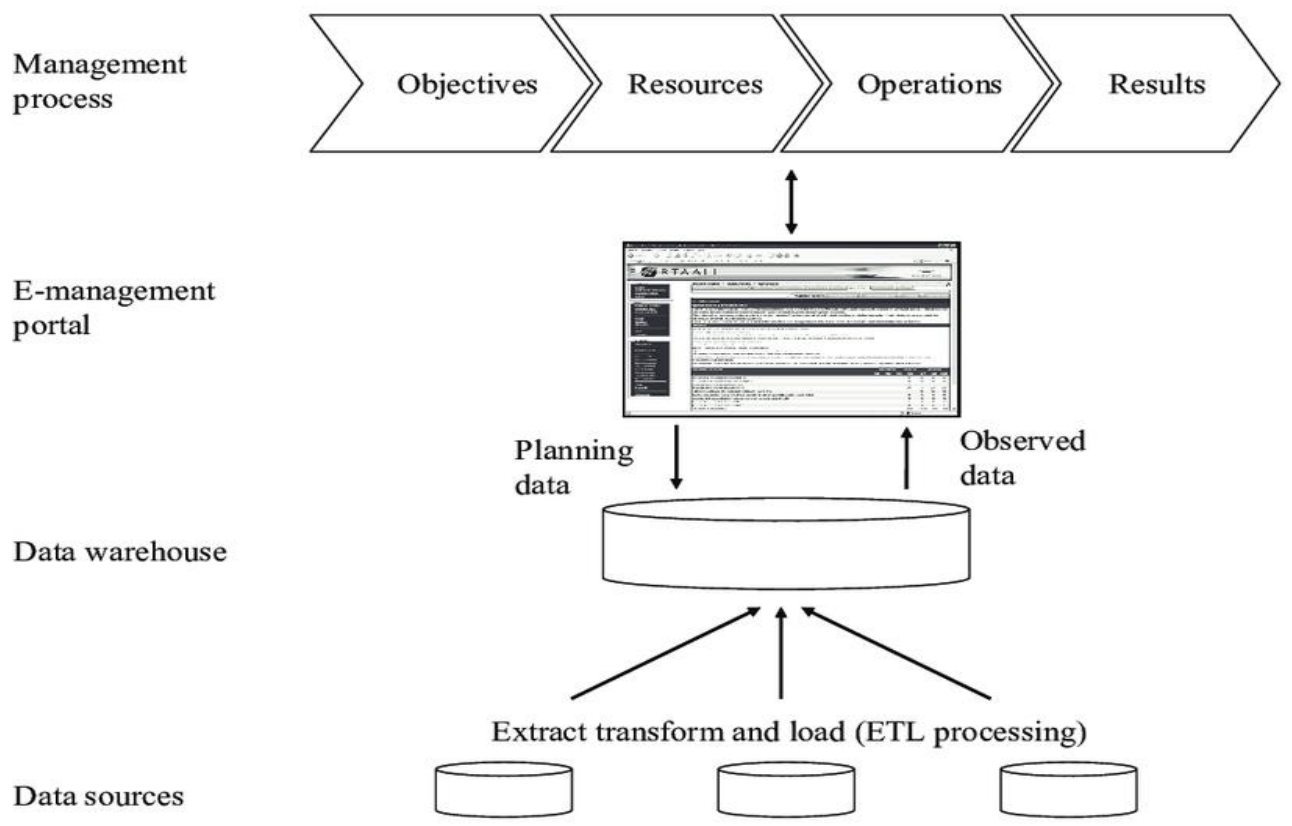

Figure 2 Architecture of the EMIS

Source: Kettunen, Juha. (2008). Management Information System in Higher Education 


\section{The characteristics of EMIS in a systemic skills environment Timeliness and reliability}

Up-to-date data and information development require that all prospective data and information manufacturers, customers, or customers have mutual knowledge of the following:

1) EMIS data generated frequently must satisfy the requirements of the general instructional planning and budgeting process.

2) Regularly generated EMIS information must satisfy the requirements of educational services such as the Logistics Unit and other classroom supply systems.

3) EMIS information generated regularly must fulfill the requirements of instructional surveillance and assessment, as well as timely policy research and guidelines.

4) Regularly generated EMIS information must satisfy the requirements of global cooperation and communication.

The timeliness within the Ministry of Education to meet these requirements is crucial to the achievement of EMIS growth. Obsolete data may not have much significance for use even after it has been generated, leading in missed possibilities for action and widespread distrust of information customers within or outside the organization. The method of data collection, data entry, data processing, data integration, data analysis, and data reporting should be brief, effective, and productive in order to ensure the prompt output of data and information to satisfy these requirements. This can often be accomplished by raising the amount of effort, starting previous preparations, proposing and enhancing task deadlines, institutionalizing EMIS as a routine management process, and enhancing EMIS datarelated operations ' coordinating ability. Reliable data generation indicates that once generated, EMIS information must reliably report the nation, district, or school's "present reality or status" or "trend of change" in academic growth. It implies that policymakers, planners, budget makers, field instructional officers, directors, educators, parents, and learners can trust the sources of information and information. To gain such confidence, it is necessary to treat information collection as a scientific process of finding facts. Variables must be indicative in evaluating meaningfully certain aspects of the education scheme or sub-systems. Whether information collection is routine or ad hoc, data collectors need to be well trained and ready to follow strict scientific measures. They should not be left with much flexibility in methodology interpretation or the liberty to alter the information collection process.

Nearly all aspects of data and information manufacturing processes can affect the level of data reliability, including the design of data collection tools, clarity of question items, field data collection techniques, academic and ethical level of participants, computer database applications design, data entry processes, data aggregation techniques, data integration processes, and

The general layout of the information collection method, information instrument development, and computer database application design and 
development must be thoroughly designed to increase data reliability. No quantity of technological innovation can improve from initial information of lousy quality.

In data and information management, "Garbage in, garbage out" is as valid as it is in computer programming. Timeliness and reliability can influence the amount of confidence and trust in the data of the information consumer. Delay in information manufacturing and unreliable information output can readily lead to a lack of frustration in information use and management, leading in inadequate planning and budgeting, tracking and assessment, policy analysis, and decision making.

When consumers of data and information (e.g., policy makers, analysts) lose faith in the capacity or credibility of EMIS, they often prevent assistance to maintain, reinforce, and upgrade the EMIS scheme. In turn, manufacturing capacity for data and information becomes even worse or decreased, further jeopardizing the ability to generate timely and reliable data. It is necessary to eradicate this vicious cycle that permeates specific instructional systems. A good culture of decision-making and governance based on information must be nurtured and created, allowed, and endorsed by an information-user-demand-driven EMIS (Hua and Herstein, 2003).

Integration and sharing

Integration of data is one of the most significant approaches for EMIS growth. It implies multi-source information (payroll, accomplishment, school census), multi-year, and multi-level information (student, teacher, or school level) can be connected, incorporated, or combined. Data integration aims to add value to the information already gathered and accessible within the same scheme in different scattered locations. Data integration is a must before conducting a high-level and high-quality policy analysis or planning exercise by an instructional policy analyst or planner. It is prevalent to see multiple units collecting and managing big databases within a Ministry of Education and not sharing them. These different information sets are gathered to define certain system aspects. For instance, in a Ministry of Education, information on student accomplishment is often gathered, managed, and accessible in an exam unit; teacher qualification and wage information are in a payroll office; information on enrolment and school inputs are in a statistical unit, and information on textbook supplies, classroom equipment, and other teaching resources are often in a supply office. For their task planning and management, these offices often have distinct databases and rarely share them with other offices. These various information sets are often intended in distinct apps for databases, arranged in distinct platforms, and coded with a self-developed identification code. As a result, unless a data integration strategy is implemented, data cannot be easily incorporated or used like this. There can be no monitoring and assessment system, a planning and policy analysis system, or an efficient and policy-relevant EMIS system without coordinated leadership. Without such schemes, policy investigations such as: how much teacher qualification and salary contribute to student learning accomplishment, given that the classroom climate and distribution of resources are the same? What is the effect on student learning success of a brand-new teacher training program or curriculum (or any recent instructional investment project)? We need to incorporate 
multi-source information so we can do the correct data analysis to answer the correct policy questions. Once centrally embedded and structured, multi-level information from various sources and years could have tremendous importance for policyrelevant research and evaluation and education leadership enhancement (Hua and Herstein, 2003).

\section{Effective usage}

An institutional culture of making policy choices based on data and information is one of the most critical variables contributing to the achievement of EMIS growth. This culture is a user-demand-enabling environment in which it is possible to build, strengthen, and further develop the ability for policy studies and analysis. Data and information consumers are policy makers, planners, policy analysts, and other significant stakeholders. The demand for data and information use should foster and nurture the remarkable growth of a culture of decision-making based on the information and the EMIS scheme. The institutional demand for data and information use is often converted into or proved through the ability of the Departmental Monitoring and Evaluation Unit, Policy Research and Analysis Unit, and Budgeting and Planning Unit. A weak capability would hurt the growth of EMIS in any of these leadership units.

\section{Challenges in EMIS implementation}

According to Abdul-Hamid et al., 2017, the main challenges in EMIS implementation can be wrapped up in the following categories:

- Leadership challenges

- Data challenges

- Operational challenges

- System and Technological Challenges

The precise definition of an EMIS is missing among decision-makers. Policymakers have no clear understanding of its use and general goal. There was no consensus on whether the EMIS was intended to gather and report statistical data to guide informed policymaking or whether it was merely a mechanism for storing information. The absence of a clear definition for most nations was partially linked to the significant amount of complicated departments engaged in the process - for instance, education, planning, and finance, science and technology, facilities without any single entity assuming a dominant management position. Often there was also a lack of political buy-in, which rendered the application of EMIS unsuccessful. The absence of clarity about the EMIS in some instances arose from the absence of a culture driven by data. (Abdul-Hamid et al., 2017).

Communication is often uncoordinated or non-existent within the Ministries of Education. Disjoined, semi-autonomous units working independently of each other without centralized leadership result in the absence of internal communication within the Ministry and allow data and information to be controlled by people or units. This affects the Ministry's general institutional culture and often leads to bad internal communication as well. Part of the data use culture is based on the concept 
of exchanging and communicating data. The lack of engagement with this concept also hinders efficient system management. Data and information are seen as a commodity in many locations. For example, if a data user needs information about teacher wages, the person in the payroll department who is "in charge" of this data is in an advantageous position because he has something in demand. In his view, sharing and disseminating these data and information freely is counterintuitive because once he ceases to be the sole proprietor of the data, it ceases to have any significance for him. The problem here is one of the absences of engagement to data integration and information use concepts. In order to generate significant policy indicators, data must be shared before it can be incorporated.

Moreover, it can only then be used to create better choices. The more data that is shared and used, the more important it may become, both systemically and collectively, not individually. The general advantage of the scheme is often not seen by information executives and information consumers because they are too worried about their advantages. This short-sightedness must be overcome by establishing an organizational vision for EMIS based on EMIS staff's visions, as well as by training and awareness campaigns accompanied by strategies and protocols to ensure the sharing of data and information (Hua and Herstein, 2003).

Availability of reliable and consistent data remained a concern for most projects. Countries placed too much emphasis on collecting data without focusing on the reliability and applicability of the data. As a result, considerable inconsistencies were found in the data. Even when efforts were made to produce quality data, timeliness remained a severe issue. Much time lags occurred in the publication and dissemination of information, thereby reducing the utility of data for decision making. Major causes for the failure were the inability of the government to understand the definition of EMIS, its objectives, and its usefulness in decision making (Abdul-Hamid et al., 2017).

Low technical capacity for data collection and analysis also made it challenging to produce quality data.

Quality data issues could be further categorized into three areas:

- Unreliable and poor quality of data

- Untimely production and dissemination of data

- Limited use of data for decision making

In order to facilitate and build an efficient EMIS scheme and capability, and to generate a high demand for the use of data and information from EMIS, it is necessary to strengthen organizational ability, precisely the capacity for budgeting, planning, tracking, evaluation, and policy research and evaluation and policy formulation. To assess whether an EMIS is effective or not, it must be determined whether it does so in a timely and reliable way:

- Defining, collecting and processing instructional information and statistics;

- Integrating information from various sources, various years and various academic levels within and outside the Ministry of Education;

- Systematically storing and managing databases and rapidly collecting information.

$140 \quad$ Review of International Comparative Management $\quad$ Volume 20, Issue 2, May 2019 
- Provide an annual statistical report on the present circumstances of the education scheme and comply with ad hoc data and statistical demands from the Ministry of Education's senior leadership and all other users of education information.

- Respond to and support inquiries and demands from instructional policy scientists, analysts, planners, and other management staff to support operations such as indicator growth, statistical analysis, budgeting and planning, registration projections, instructional efficiency surveys, and other quantitative system analysis and tracking and assessment (Hua and Herstein, 2003).

Designing an EMIS is a challenging task requiring financing to develop and implement a new information system at both local and national levels. For most projects, this has been a significant challenge. At the local level, problems arose in installing the computer equipment and developing the software for an information system. In many cases, the technical specifications for the development of the software were not finalized. The effort lacked coordination and failed to make the specifications compatible with the existing information systems. In some cases, the procurement process for computer equipment failed. Even when the software was developed for all levels of the education system and made available to all schools, the computerized student record system is used only partially.

Developing the EMIS and expanding the capacity of the system can be a technologically challenging task. The systems and software need to be continuously updated to accommodate the ever-changing need for data. It is also vital to ensure that information systems interact with one another to enable the integration of these systems into one comprehensive EMIS. Technical problems associated with any of the above may impair the system's functioning, such as a full breakdown or failure of an EMIS due to system capability problems, software problem, integration problem, or lack of Internet/computers to make data available to the public. (AbdulHamid et al., 2017).

\section{Conclusions}

While we face the certainty of many operations essential to the automation of today's workforce in the future and fear of job losses, we hear the loud voice of employers saying that already today there are not enough qualified employees to satisfy employers ' requirements worldwide. Interestingly enough, the types of abilities most required are overwhelmingly united, and they not just are traditional professional abilities, but are competence in cooperation, communication and mental agility tasks. On the positive hand, over the last decade, attempts to reform worldwide schooling are doing more to promote kid and young people's growth to enhance their core values and life skills. On the adverse hand, reforms of education and practice have not yet been aligned with the aforementioned activities and employers have been comparatively absent from the reform of education policy. It is therefore urgent and crucial that attempts to reform and innovate education are connected and aligned with employee gaps and needs. 
Data collection is the main component of tracking progress challenge, particularly about SDG 4. More importantly, however, a powerful EMIS also needs to make excellent use of the resulting data, generate the indicators appropriate to domestic planning, and implement the necessary education policies (Montoya, 2018).

Development of an information system for education management is crucial in modern education system management. It is intended to assist in decision-making procedures based on data. Computer technology, database instruments, and technical abilities provide the educational system with the necessary support in data and information manufacturing ability. An EMIS unit, however, requires a clear vision to see and understand what to create, whom to sustain the product, and which departments and units to include. EMIS creation includes more nurturing a new culture of leadership than setting up a data and information system. The method of data collection, integration, evaluation, and dissemination is crucial, but more critically, it is the culture of data sharing, information use, and organizational management that contributes to EMIS growth efficiency.

It is essential to realize that the growth of EMIS is not the growth of IT. No one wants "policy talk." Evidence or evaluation of the proof must support policy. To provide this proof, EMIS is being created. The findings of the analysis of this proof should be provided by EMIS and other management tasks such as surveillance and assessment, policy research and analysis, and joint budgeting and planning. This is the assistance that education ministries and policymakers in education ministries deserve (Hua and Herstein, 2003).

\section{References}

Abdul-Hamid, H. 2014, What Matters Most for Education Management Information Systems: a Framework Paper, World Bank, [Online] Available at http://wbgfiles.worldbank.org/documents/hdn/ed/saber/supporting_doc/Bac kground/EMIS/Framework_SABER-EMIS.pdf [Accessed 10 April 2005].

Abdul-Hamid, H., Namrata S., and Mintz S., 2017, Lessons Learned from World Bank Education Management Information System Operations: Portfolio Review, 1998-2014, World Bank Studies, Washington

Hua, H. and Herstein, J., 2003, Education management information system (EMIS): Integrated data and information systems and their implications in educational management. In Annual conference of comparative and International Education Society, [Online] Available at http://www.infodev.org/infodev-files/resource/InfodevDocuments_188.pdf [Accessed 10 April 2005].

Kettunen, J., 2008, Management Information System in Higher Education, [Online] Available at https://www.researchgate.net/publication/264552945_ Management_Information_System_in_Higher_Education [Accessed 15 May 2019]

Makwati, G., Audinos B., and Lairez B., 2003, The Role of Statistics in Improving the Quality of Basic Education in Sub-Saharan Africa., Working Document, 
Association for the Development of Education in Africa, African Development Bank, Tunis, Tunisia.

Montoya, S., 2018, Why We Need Effective Education Management Information Systems, [Online] Available at http://uis.unesco.org/en/blog/why-we-needeffective-education-management-information-systems [Accessed 15 May 2019]

UNESCO, 2003, Information tools for the preparation and monitoring of education plans, Education policies and strategies 5.

UNESCO, 2018, Re-orienting Education Management Information Systems (EMIS) towards inclusive and equitable quality education and lifelong learning, [Online] Available at http://unesdoc.unesco.org/images/0026/002619/ 261943e.pdf [Accessed 15 May 2019]

Wako, 2003, Education Management Information Systems (EMIS), Harare, Fontline Electronic Publishing.

World Bank, 2011, Learning for All: Investing in People's Knowledge and Skills to Promote Development; World Bank Group Education Strategy 2020, World Bank, Washington, DC. 